Bull. Mater. Sci., Vol. 15, No. 1, February 1992, pp. 47-54. (C) Printed in India.

\title{
Earthquake hazards for cross-country pipelines
}

\author{
PRADIPTA BANERJI \\ Department of Civil Engineering, Indian Institute of Technology, Powai, Bombay 400076, \\ India
}

\begin{abstract}
A review of the current state-of-knowledge in risk-based earthquake-resistant design of pipeline systems is presented. Damage to pipelines during past earthquakes is studied, and the necessity of evaluating the risk to such systems from earthquakes for their economic design is illustrated. The various aspects of seismic risk analysis of pipeline systems are briefly studied and a conclusion reached that given a tectonic and seismic data for the region in which pipelines lie, in addition to proper definition of various levels of pipeline unserviceability, it is possible to estimate the earthquake hazards to such pipelines with a reasonable degree of accuracy. Since this subject is of topical interest to this country, with increasing number of pipeline systems laid in earthquake-prone areas, specific areas are identified where more information is required before meaningful seismic risk analysis of pipelines can be done in India.
\end{abstract}

Keywords. Pipelines; lifelines; earthquake hazards; seismic risk; damage; reliability.

\section{Introduction}

Past experiences--San Francisco (1906), Tokyo (1923), Niigata (1964), Alaska (1964), San Fernando (1971), Managua (1972), Coalinga (1983) and Loma Prieta (1989)show that long utility pipeline networks have been extensively damaged during severe earthquakes, with catastrophic consequences on the communities where these occurred (Katayama et al 1975; Ariman and Muleski 1981; Kamada et al 1981). This is because it is precisely in the aftermath of a devastating earthquake that the serviceability of these utility pipelines is essential, for preventing fires due to cracked gas lines, aiding fire-fighting, and keeping underground communication systems working. In India, it is probably fortunate that most of the recent earthquake devastation occurred in relatively rural regions, where no pipeline damage has been reported. However, with rapid improvement in provision of utility pipeline networks in urban areas, and construction of cross-country transportation pipelines- $\mathrm{HBJ}$; Assam-Barauni; proposed Kandla-Bhatinda - through seismic zones of the country, the likelihood of pipeline damage in future earthquake increases significantly.

Thus, in regions that have had moderate to high number of earthquake occurrences in the past, or are deemed to be geologically unstable with possible future earthquakes likely, pipelines should be designed to be earthquake-resistant. But it is not practically and economically feasible to design these pipelines to sustain no damage at all during a major earthquake. The practical problem is that there is always an uncertainty in the estimation of the maximum earthquake response of a pipeline during its expected operating life due to the randomness of earthquake occurrence and variability in induced ground motions. Therefore, an optimum design is needed that balances the costs of construction and possible repair on the one hand, and the acceptable losses of performance during earthquakes on the other. To lay the basis for this optimum earthquake-resistant design of a pipeline system, the probable earthquake hazards 
for the system during its lifetime can be assessed through a seismic risk analysis of the system. The problem is complex, and is made even more so by the different types of material-metal (cast iron, stainless steel, carbon steel), reinforced concrete, prestressed concrete, plastic (polyethylene), vitrified clay, and PVC - used in pipeline construction. This is because each construction material has its own response characteristics and damage potentials, which affect the earthquake risks associated with the pipelines. However, the assessment of seismic risk of pipeline systems has been receiving considerable attention (ASCE 1977, 1981; Shinozuka et al 1979) since the 1971 San Fernando earthquake, during which the seismic hazards for pipelines were glaringly illustrated (Ariman and Muleski 1981). Based on these studies guidelines for earthquake-resistant design of pipeline systems have also been suggested (Hall and Newmark 1978; ASCE 1984).

It is the intention of the author to present in this paper a brief review of the current state-of-knowledge in seismic risk assessment of pipeline systems, and the areas where significant study is required to make the presently available analytical tools applicable to realistic risk assessments in the Indian context (Banerji and Datta 1989).

\section{Earthquake damage in pipeline}

For a realistic seismic risk assessment of a pipeline system it is important that the failure and unserviceability criteria used in the risk analysis reflect the actual behaviour of and damage to a pipeline during an earthquake. Therefore, it is instructive to review the different types of damage that have been observed in pipeline networks after the occurrence of earthquakes in the past, and decipher the causes of these damages.

There are three different types of earthquake damage to pipeline systems that have been observed in the field (Ariman and Muleski 1981; Shinozuka et al 1979): (i) yield or rupture of the pipeline; (ii) pull-out of pipeline due to failure of joint; and (iii) buckling of pipeline.

The first two types of failure, namely, yield or rupture of the pipeline, and failure of joints, occur mostly due to large soil displacements or variation of ground motion along the length of the pipeline. These are caused due to either one or a combination of the following factors: (a) severe ground motion due to proximity of pipeline to earthquake source; (b) spatial variation of ground motion due to seismic wave travelling through the soil medium as a body wave (longitudinal or shear), or at the free surface as a surface wave (Rayleigh or Love); (c) fault rupture and movement; (d) landslides occurring on slopes; and (e) liquefaction of the supporting soil medium due to it consisting of a substantial volume of fine-grained, loose, saturated sand, and the earthquake ground motions being of high intensity and long duration.

Buckling of pipelines mainly occurs due to excessive axial deformations in the pipeline segments caused by differential longitudinal displacements along the members. This type of damage occurs mostly when pipelines supported by a firm soil medium run into a horizontally adjacent layer of soft soil, where in the transition region relative movements are caused by the difference in dynamic properties of the two soil layers. 


\section{Aspects of seismic risk analysis}

Assessment of seismic risk of a pipeline system requires consideration of the following aspects of the problem (see figure 1): (i) determination of the earthquake hazards for the area covered by the pipeline system; (ii) estimation of the reliability, or conversely the vulnerability, of pipeline components at different damage levels to these earthquake hazards by analysis of their structural behaviour during the earthquakes; and (iii) evaluation of the seismic risk to the system, for different performance levels, by combining appropriately the vulnerability of the critical components of the system. Each of these three aspects have to be realistically characterized for the risk analysis to be meaningful. Each source of earthquake hazard in the pipeline area has to be identified, and the hazard due to that source has to be quantified. The actual damage to pipeline components from each hazard source has to be idealized, and an estimate of the probability of damage occurring has to be obtained. For evaluating the system
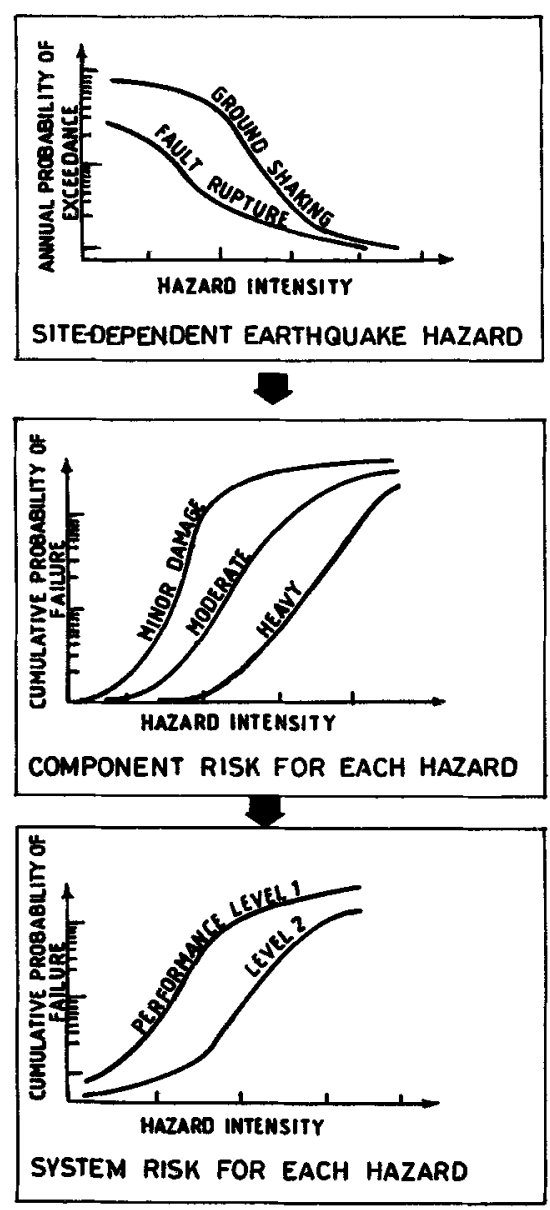

Figure 1. Various aspects of seismic risk assessment of pipeline systems. 
risk it is necessary to idealize the pipeline system network, and have some correlation between the idealized performance level and the actual degree of unserviceability. Furthermore, the spatial variation along the network of soil conditions and earthquake ground motions should also be considered. At present, however, various aspects of the problem have not yet been properly understood and therefore cannot be incorporated into any rational analysis.

\subsection{Earthquake hazards}

Proper characterization of the earthquake hazards for an area covered by a pipeline system is one of the most difficult aspects of risk assessment of such systems. This is because of the variety of sources of these hazards: severe ground motion, spatial variation of ground motion due to the travelling seismic waves, fault movement, soil liquefaction and landslides. Of these, an extensive effort has been made on characterization of hazards due to only the first two sources (Shinozuka et al 1979; Kamada et al 1981). Some studies have been carried out to determine the liquefaction potential of soil deposits in some areas, but no comprehensive recommendations exist at present. Almost no work is available for characterizing hazards due to fault movements or landslides. Some general guidelines for estimating the seismic hazard of an area covered by a pipeline system are provided by ASCE (1984). In this paper only the characterization of earthquake hazards due to ground motion and its spatial variation will be discussed.

The earthquake hazard at a site due to ground motion is defined as the probability that a ground motion intensity parameter $Y$ will exceed a specified threshold value $y_{0}$ during a certain time period (usually a year), (see figure 1) i.e.

$$
\text { hazard }=P\left(Y \geqslant y_{0}\right)
$$

or by the return period $T_{y_{0}}$ of a ground motion intensity of $y_{0}$, i.e.

$$
\text { hazard }=T_{y_{0}}=1 /\left[P\left(Y \geqslant y_{0}\right)\right] \text {. }
$$

Various ground motion intensity parameters have been defined by past researcherspeak ground acceleration, peak ground velocity, r.m.s. ground acceleration, site intensity on the Modified Mercalli Intensity (MMI) scale, and Arias intensity - but the most common are the peak ground acceleration and site intensity on the MMI scale, since they have been historically used to characterize effects of earthquakes.

To determine the aforementioned exceedance probabilities or return periods in the pipeline network area the following information is required: (a) regional tectonic data that provide estimates for earthquake source mechanism parameters such as focal depth, location and orientation of causative faults, and magnitudes of earthquakes; (b) historical seismicity data that allow development of a probability distribution for earthquake magnitudes on each fault or source and estimation of relative seismic activity in different sources in the region; and (c) a set of attenuation laws specific to the local geological condition of the region that relate ground motion intensity parameters at a site to earthquake magnitude and distance from source. Using this information the seismic hazards in the area of the pipeline system are estimated through the process of microzonation, where the area is divided into zones based on geological, tectonic, and historical seismicity information. 
Unfortunately, except on the West Coast of North America, Japan, and recently Taiwan, there is serious lack of consistent field data on earthquakes. This could possibly be overcome with the establishment of several dense strong motion arrays in regions where significant seismicity has been historically present, or tectonically probable. In India, the major hurdle to be overcome remains the almost total lack of available seismicity data. Recently, however, there have been some efforts to establish arrays in the Terai and Shillong Plateau regions. In spite of this, to the best of the author's knowledge, there has been very little effort directed towards estimating earthquake hazards in different regions of the country through microzonation, and this remains an area where significant amount of work is required.

\subsection{Component vulnerability analysis}

After earthquake hazards in the pipeline system area are characterized, the next step in risk assessment of this system is estimating the reliability, or conversely the vulnerability, of the system components to these hazards. The reliability of a component is measured by the probability of survival of that component when subjected to a specific source and intensity of earthquake hazard, whereas the vulnerability is measured by the probability of component failure. Since failure definition depends on the damage state corresponding to a specified level of component unserviceability, it follows that the reliability/vulnerability of a component is also a function of the damage state. Therefore, mathematically speaking

$$
\begin{aligned}
& \text { vulnerability: } p_{f} \text { (damage state, hazard intensity), } \\
& \text { reliability: }\left[1-p_{f}\right. \text { (damage state, hazard intensity)], }
\end{aligned}
$$

where $p_{f}$ denotes the probability of failure. A reliability/vulnerability analysis of a pipeline component involves the estimation of this probability of failure for a variety of damage states and hazard intensities from each hazard source (see figure 1). There are three distinct methodologies available in literature for vulnerability analysis of pipeline components: qualitative, empirical, or analytical.

The simplest vulnerability analysis method consists of a qualitative assessment of pipeline component vulnerability (Eguchi 1983). A break in the pipeline constitutes failure and this probability of failure for each earthquake hazard (ground shaking, fault rupture, liquefaction, landslides) intensity is estimated to be high, moderate, or low from previous experience. An example of a qualitative vulnerability analysis for underground pipeline components is presented in table 1 . This example illustrates the importance of the material used for pipeline construction and the type of joints in determining the vulnerability of pipelines to earthquake hazards. Since mild steel pipes with arc-welded joints perform the best irrespective of source and intensity, it can be concluded that pipelines and joints should be made of ductile material, like mild steel, to reduce risks of seismic failure.

The problem with qualitative risk assessment methods lies in their essential simplicity. From qualitative probability of failure estimates it is rather difficult to evaluate the system risks. It is this weakness of the method that leads to the next level of methods, empirical vulnerability analyses that give quantitative estimates of probability of failure. These methods are also based primarily on statistical observations from past earthquakes, and till recently constituted the best way of estimating the vulnerability 
Table 1. Qualitative vulnerability analysis for underground pipelines (after Eguchi 1983).

\begin{tabular}{lccccccc}
\hline & $\begin{array}{c}\text { Ground motion } \\
\text { intensity (MMI) }\end{array}$ & & \multicolumn{2}{c}{$\begin{array}{c}\text { Fault rupture } \\
\text { displ., cm }\end{array}$} & & \\
\cline { 2 - 3 } Pipe type & VIII & X & & 25 & 250 & $\begin{array}{c}\text { Liquefaction } \\
\text { lurching }\end{array}$ & Landslide \\
\hline WSAWJ & L & L & L & L & L & L \\
WSGWJ & M & H & H & H & H & H \\
CI & M & M & H & H & H & H \\
AC & M & M & H & H & H & H \\
\hline
\end{tabular}

Vulnerability levels:

Low $<0.1$ breaks per km; Moderate 0.1 to 1 breaks per $\mathrm{km}$; High $>1$ break per km. Pipe types:

WSAWJ, welded-steel with arc-welded joints; WSGWJ, welded steel with gas-welded joints; CI, cast iron; AC, asbestos cement
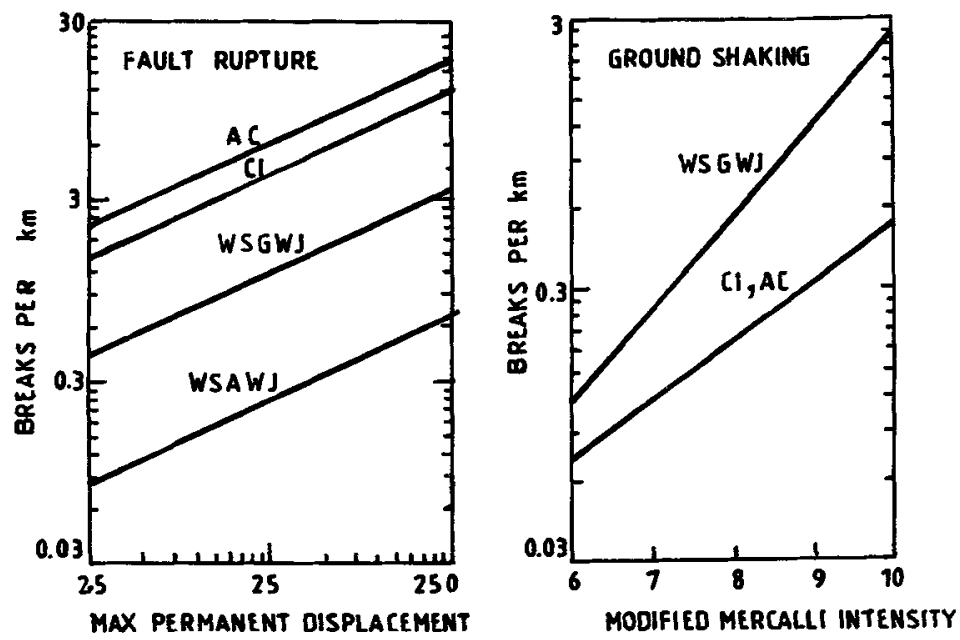

Figure 2. Empirical vulnerability analysis for underground pipelines (after Eguchi 1983). See table 2 for notations.

of pipeline components. These methods essentially correlate the observed percent damage or failure rate in the pipeline due to each source of possible earthquake hazard (Ariman and Muleski 1981; Eguchi 1983). An example of empirical analysis for underground pipelines is presented in figure 2 , where again a break in the pipeline constitutes failure. The major problem with these methods is that this type of analysis gives an average representation of the vulnerability of pipeline components. For example, it has been established that the reliability of a pipe to any earthquake hazard depends on its diameter, but empirical analysis methods cannot reflect this. Despite these obvious shortcomings, these methods provide an excellent basis for more refined analytical reliability analysis.

Analytical reliability analysis methods provide the most detailed characterization 
of the vulnerability of pipelines to earthquake hazards. These methods require an idealization of the pipeline components, e.g. each segment is idealized as an assemblage of beam or shell elements, connected to other segments through flexible joints, which are idealized as systems of springs and dashpots (Ariman and Muleski 1981; Shinozuka et al 1979). A stochastic analysis of the response of these idealized structures is carried out, and standard reliability methods are used to evaluate the probabilities of failure of a component for a defined damage state. The main concern in these methods is accurate representation of actual component serviceability by idealized performance functions that can be related to the response of the system when subjected to a particular earthquake hazard. Good correlation between idealized damage states and actual serviceability conditions ensures reliable estimates of the component vulnerability.

\subsection{System vulnerability analysis}

There are two distinct approaches of determining the vulnerability or risk of a pipeline system, though both use the estimates of the vulnerability of each pipeline component. One approach estimates a probability of system failure through the use of simulation techniques. The other approach estimates the same system vulnerability measure through the use of rigorous mathematical system network analysis techniques. However, both require the definition of system performance criteria that depend on individual component performance criteria and correlate well with actual pipeline system serviceability conditions.

In the former approach, the probability of system failure corresponding to each system performance state is obtained statistically from the simulation, using techniques such as Monte Carlo simulation (Shinozuka et al 1979) of a large number of system states. Each system state is simulated by considering each component state to be given by a binary random number that is generated from the estimate of the component probability of failure. Then the system is analysed to check whether it remains functional or is unserviceable (given a particular performance criterion). After a large number of such simulations have been done the probability of system failure (again for a given performance criterion) can be estimated from the ratio of the number of failure states obtained to the total number of simulations carried out.

In the latter approach, the pipeline system failure probability is obtained from a combination of the individual component failure probabilities using systems reliability algorithms (Whitman and Cornell 1976; Taleb-Agha 1977). There are various techniques available, e.g. cut-set approaches, fault-tree analyses, etc. each with its different set of limitations and assumptions, the discussion of which is beyond the scope of this paper.

Computationally, the analytical approach is far more efficient than the simulation approach, and seemingly more elegant. However, for some definitions of system performance criterion that involve flow rates in pipelines, analytical procedures do not give reliable estimates of the system vulnerability, because the simplified idealizations that have to be made to apply these methods do not correlate well with the actual physical state. In these situations, only simulation approaches give proper estimates of the system risk. 


\section{Conclusions}

Given restrictions on the length of the paper, a fairly comprehensive albeit brief study of the various aspects involved in assessing the earthquake hazards for pipeline systems has been attempted. The various methods that are available for estimation of the hazards have been outlined.

In India, at present, due to the paucity of observed pipeline damage, it is suggested that seismic hazards for pipeline systems be assessed only by application of the analytical procedures outlined here. Available relationships between earthquake hazards at site and ground motion estimates in literature may be used, although it must be realized that these are approximations at best that should be correlated to actual field data as it becomes available, and changed if necessary. To reduce the risk to pipeline systems from earthquake hazards due to sources other than severe ground motion the following recommendations are made (Ariman and Muleski 1981; ASCE 1984):

- locate pipelines, whenever possible, away from active faults, steep hillsides, and soft soil sites;

-increase flexibility of the system through use of more ductile material and flexible joints;

- provide "fail-safe" systems at sites where damage may be anticipated.

It is suggested that work in the following areas needs to be carried out in this country before proper estimates of seismic risk to pipeline networks can be obtained, which lead to their economic risk-based earthquake-resistant design:

-collecting and interpreting data from currently available strong-motion arrays;

- setting up strong motion arrays in regions of strong seismicity;

- evaluating earthquake hazards in different seismic zones of the country;

- evaluating site-specific earthquake hazards through the process of microzonation.

\section{References}

ASCE 1977 The current state of knowledge of lifeline earthquake engineering, American Society of Civil Engineers, New York

ASCE 1981 Lifeline earthquake engineering: The current state of knowledge, American Society of Civil Engineers, New York

ASCE 1984 Guidelines for seismic design of oil and gas pipelines, Technical Council on Lifeline Earthquake Engineering (Gas and Liquid Fuels Committee), American Society of Civil Engineers, New York

Ariman T and Muleski G E 1981 Int. J. Earthquake Engg Struct. Dyn. 9133

Banerji P and Datta T K 1989 Seismic response and risk analysis of pipeline systems, Research Proposal submitted to the Department of Science and Technology, Government of India

Eguchi R T 1983 Seismic vulnerability models for underground pipes, Proc. Int. Symp. Lifeline earthquake engineering, (Portland: ASME) pp 82

Hall W J and Newmark N M 1978 J. Tech. Councils ASCE 10491

Kamada H, Goto H, Sugito M and Asaoka K 1981 Seismic risk and performance of water lifelines, Proc. Symp. on Probabilistic Methods in Structural Engineering, (New York: ASCE) pp. 179

Katayama T, Kubo K and Sato N 1975 Earthquake damage to water and gas distribution systems, Proc. US National Conference on Earthquake Engineering, (Ann Arbor: EERI) pp 396

Shinozuka M, Takada S and Ishikawa H 1979 J. Pressure Vessel Technol. 10131

Taleb-Agha G 1977 Bull. Seismol. Soc. Am. 671625

Whitman R V and Cornell C A 1976 Seismic risk and engineering decisions, (eds) C Lomnitz and E Rosenblueth (New York: Elsevier) ch. 9, p 339 